\title{
Circ- 104916 is downregulated in gastric cancer and suppresses migration and invasion of gastric cancer cells
}

This article was published in the following Dove Press journal:

OncoTargets and Therapy

17 July 2017

Number of times this article has been viewed

\author{
Jin Li* \\ Li Zhen* \\ Yan Zhang* \\ Liying Zhao \\ Hao Liu \\ Danxian Cai \\ Hao Chen \\ Jiang Yu \\ Xiaolong Qi \\ Guoxin Li
}

Department of General Surgery, Nanfang Hospital, Southern Medical University, Guangzhou, People's Republic of China

*These authors contributed equally to this work
Correspondence: Guoxin Li; Xiaolong Qi Department of General Surgery, Nanfang Hospital, Southern Medical University, 1838 North Guangzhou Avenue, Guangzhou 510515, People's Republic of China

Tel +86206164 I68I

Fax +862061641683

Email gzliguoxin@163.com; qixiaolong@vip.163.com
Abstract: Circular RNAs are a large class of noncoding RNA that have shown huge capabilities as gene regulators. Recent evidence suggest that circular RNAs are associated with many diseases, especially cancer. However, little attention has been focused on the expression and function of circular RNA in gastric cancer (GC). In this study, we demonstrate that the expression of circ-104916 is downregulated in GC tissues and cell lines. A lower expression of circ-104916 appeared in deeper invasion depth, higher tumor stage and more frequent lymphatic metastasis patients. Overexpression of circ-104916 effectively inhibited the proliferation, migration and invasion abilities of GC cells in vitro. Western blot showed that circ-104916 overexpression upregulated E-cadherin and downregulated N-cadherin, Vimentin and Slug, indicating that circ104916 was involved in the epithelial-mesenchymal transition process. Our results revealed that circ-104916 might be a novel potential tumor suppressor and biomarker of GC.

Keywords: circular RNA, noncoding RNA, circ-104916, gastric cancer, EMT

\section{Introduction}

Gastric cancer (GC) is the third leading cause of cancer death worldwide, which affects $>400,000$ patients/year in China. ${ }^{1}$ More than $80 \%$ of the Chinese patients were diagnosed at advanced stages, on which surgery with curative intent required extended lymphadenectomy. Laparoscopic distal gastrectomy had been proved to be safe for advanced GC. ${ }^{2}$ Even with advanced systematic therapy, the 5-year overall survival of patients with late-stage GC was not $>20 \%{ }^{3}$ Therefore, it is of great significance to investigate the mechanism of GC progression and metastasis.

Circular RNA (circRNA) was first detected over 2 decades ago, which was considered a byproduct of splicing errors. ${ }^{4}$ With the development of high-throughput RNA sequencing and bioinformatics, it was clear that circular RNA was abundant and stable in eukaryotic cell. ${ }^{5,6}$ Moreover, studies had found out that circular RNA expression was dysregulated in pancreatic cancer, ${ }^{7}$ ovarian cancer, ${ }^{8}$ bladder cancer ${ }^{9}$ and hepatocellular cancer. ${ }^{10}$ CircRNA could act as microRNAs sponge to regulate gene expression. The most famous circular RNA was CDR1as, which contained $>70$ conventional binding sites and functioned as a super sponge for miR-7. ${ }^{11}$ Recent studies showed that CDR1as was a risk factor of hepatic microvascular invasion in hepatocellular carcinoma $(\mathrm{HCC})^{12}$ and knockdown of CDR1as suppressed the HCC cell proliferation and invasion. ${ }^{13}$ However, the expression and function of circular RNA in GC are still unknown.

In our previous study, via microarray, we found that the expression of circ-104916 was reduced in GC..$^{14}$ In this study, we found that circ-104916 was significantly 
downregulated in GC tissues by expending the sample size. In vitro studies showed that circ-104916 could suppress the proliferation, migration and invasion capabilities of GC cells. This is the first study to investigate the role of circ-104916 in GC, which suggests that circ-104916 may be a potential tumor suppressor and biomarker of GC.

\section{Materials and methods}

\section{Tissues samples}

Human samples including paired cancer specimens and adjacent normal tissues were obtained from the patients who underwent surgical resection from November 2015 to May 2016 at Nanfang Hospital, Southern Medical University. All fresh samples were immediately frozen after resection and stored at $-80^{\circ} \mathrm{C}$ until use. Tumors were staged according to the TNM staging classification of stomach carcinoma of American Joint Committee on Cancer (AJCC; 7th ed, 2010). None of the patients had received chemotherapy or radiotherapy before surgery. The study was approved by the Clinical Research Ethics Committee of Nanfang Hospital of Southern Medical University. Written informed consent was obtained from all participants.

\section{RNA extraction and quantitative real-time polymerase chain reaction (qRT-PCR)}

Total RNA was extracted from the cells and tissues using TRIzol reagent (Takara, Tokyo, Japan) according to the manufacturer's protocol. After RNA extraction, reverse transcriptase (Takara) was used for synthesizing cDNA according to the manufacturer's instructions. The expression level of the circRNAs was evaluated by quantitative PCR (qPCR) using SYBR Green assay. Specific divergent primers were designed to amplify the circular transcripts. PCR was performed in $10 \mu \mathrm{L}$ reaction volume, including $2 \mu \mathrm{L}$ of cDNA, $5 \mu \mathrm{L}$ of $2 \times$ Master Mix (Takara), $0.4 \mu \mathrm{L}$ of forward primer $(10 \mu \mathrm{M}), 0.4 \mu \mathrm{L}$ of reverse $\operatorname{primer}(10 \mu \mathrm{M})$ and $2.2 \mu \mathrm{L}$ of double distilled water. The reaction was set at $95^{\circ} \mathrm{C}$ for $10 \mathrm{~min}$ for pre-denaturation and then at $95^{\circ} \mathrm{C}$ for $10 \mathrm{~s}$ and at $60^{\circ} \mathrm{C}$ for $60 \mathrm{~s}$ repeating 40 cycles. $\beta$-actin was used as a reference. We used the following primer sequences: circ104916 - forward: 5'-GCTCGGTGACCTTGGTCTGG-3' and reverse: 5'-GCGTGTTGGGATGCCTCTGT-3' and $\beta$-actin - forward: 5'-TGGATCAGCAAGCAGGAGTA-3' and reverse: 5'-TCGGCCACATTGTGAACTT-3'. Both target and reference were amplified in triplicate wells. The relative level of each circRNA was calculated using the $2^{-\Delta \Delta C_{t}}$ method.

\section{Cell culture}

Human GC cell lines (AGS, MKN-28, NCI-N87 and MKN-45) and human gastric mucosa epithelial cells (GES-1) were propagated in Roswell Park Memorial Institute (RPMI) 1640 (HyClone, Logan, UT, USA) supplemented with 10\% fetal bovine serum (FBS; Capricorn, Ebsdorfergrund, Germany).

\section{Circular RNA plasmid construction and transfection}

We constructed the circular RNA plasmid used in this study. Human circ-104916 cDNA was synthesized by GENEWIZ (Suzhou, China) and cloned into pcDNA3.1. We used a 651-bp DNA fragment corresponding to exon 1, exons 3-6 and exon 8 of the NEK6 gene and added $1 \mathrm{~kb}$ upstream and $200 \mathrm{bp}$ downstream to the nonlinear splice sites. In addition, an 800-bp DNA stretch was added upstream of the splice acceptor site and inserted downstream in the reverse orientation. ${ }^{11}$ The GC cells were seeded at six-well plates and then transfected at $48 \mathrm{~h}$ with pcDNA-hsa-circ-104916 (4 $\mu \mathrm{g})$ or control plasmid $(4 \mu \mathrm{g})$ using Lipofectamine 3000 Transfection Reagent according to the manufacturer's protocol (Thermo Fisher Scientific, Waltham, MA, USA).

\section{Cell proliferation assay}

Cell proliferation was assayed using the Cell Counting Kit-8 (CCK-8) assay (Dojindo Laboratories, Kumamoto, Japan) according to the manufacturer's protocol. The transfected cells were plated in 96-well plates (1,500 cells/well). Cell proliferation was detected every $24 \mathrm{~h}$ according to the manufacturer's protocol. Briefly, $10 \mu \mathrm{L}$ of CCK 8 solution was added to each well and incubated for $2 \mathrm{~h}$ at $37^{\circ} \mathrm{C}$. The solution was then measured spectrophotometrically at $450 \mathrm{~nm}$.

\section{In vitro cell migration and invasion assays}

A total of $1 \times 10^{5}$ BGC 823 and MGC 803 cells transfected for $48 \mathrm{~h}$ with $4 \mu \mathrm{g}$ pcDNA-hsa-circ-104916 or pcDNA3.1 were plated in the upper chamber of transwell assay inserts (Corning Incorporated, Corning, NY, USA), which contained $200 \mu \mathrm{L}$ of serum-free RPMI 1640 with a membrane ( $8 \mathrm{~mm}$ pores) to test migration. The lower chambers were filled with RPMI 1640 containing $10 \%$ FBS. The cells on the filter surface were fixed with methanol, stained with crystal violet and photographed with a digital microscope after $24 \mathrm{~h}$. The cell numbers were calculated in five random fields for each chamber.

The transfected cells $\left(1 \times 10^{6}\right)$ were plated in the top chamber (Corning Incorporated) that was precoated with Matrigel (BD Biosciences, San Jose, CA, USA) in $200 \mu \mathrm{L}$ serum-free RPMI 1640 to test cell invasion. There was 
$750 \mu \mathrm{L}$ of $10 \%$ FBS-RPMI 1640 in the bottom chambers. The invasion function was determined after $48 \mathrm{~h}$.

\section{Western blot assay}

The cellular proteins were extracted $48 \mathrm{~h}$ after transfection. The cells in each well were lyzed in $50 \mu \mathrm{L}$ of lysis buffer containing radioimmunoprecipitation assay (RIPA; $49.5 \mu \mathrm{L})$ and protease inhibitor cocktail $(0.5 \mu \mathrm{L})$. Then, $30 \mu \mathrm{g}$ of protein was resolved by sodium dodecyl sulfate polyacrylamide gel electrophoresis (SDS-PAGE) analysis and transferred onto a polyvinylidene difluoride (PVDF) membrane. The primary antibodies used were anti-E-cadherin (1:1,000; Abcam, Cambridge, UK), antiN-cadherin (1:5,000; Abcam), anti-Vimentin (1:1,000; Abcam), anti-Slug (1:1,000; Abcam), anti-Snail (1:1,000; Abcam) and anti-GAPDH (1:10,000; Proteintech group, Inc., Chicago, IL, USA). The membranes were washed and then incubated with goat anti-rabbit horseradish peroxidase (HRP)-conjugated secondary antibody (1:10,000; Abcam) for $2 \mathrm{~h}$ at room temperature. The blots were visualized by enhanced chemilu-minescence detection.

\section{Statistical analysis}

All statistical analyses were performed using SPSS 23.0 software (IBM Corporation, Armonk, NY, USA). Data were expressed as mean \pm standard error of the mean (SEM) from at least three separate experiments. Differences between groups were analyzed using Student's $t$-test analysis. Correlation between the clinicopathological data and circ-104916 was analyzed using chi-square test. $P$-value $<0.05$ was considered as statistically significant.

\section{Results \\ Downregulation of circNEK6 in human GC}

In our previous study, the microarray results suggested that the expression of circRNAs in gastric carcinoma tissues was abnormal compared with adjacent normal tissues (Figure S1). The expression of circ-104916 was reduced in GC tissues. To confirm hsa-circ-104916 expression in GC, qRT-PCR was performed in 70 pairs of human GC specimens and their adjacent noncancerous tissue samples. More than 50\% reduction in circ-104916 expression was observed in $65.7 \%(46 / 70)$ of GC tissues (Figure 1A). The expression of circ-104916 was significantly lower in GC tissues than in the corresponding adjacent noncancerous tissues (Figure 1B). Theses results suggested that circ-104916 was downregulated in GC tissues, which was consistent with our microarray result.

\section{Correlation with the clinicopathological data and expression of circ- 104916}

To assess the coloration of circ-104916 expression with clinicopathological data, the level of circ-104916 in tumor tissue was categorized as low group or high group in relation to the media value of relative circ-104916 expression (0.36-fold, tumors/no cancers). Clinicopathological factors were analyzed in the high or low groups. The expression of circ-104916 was significantly associated with invasion depth,
A

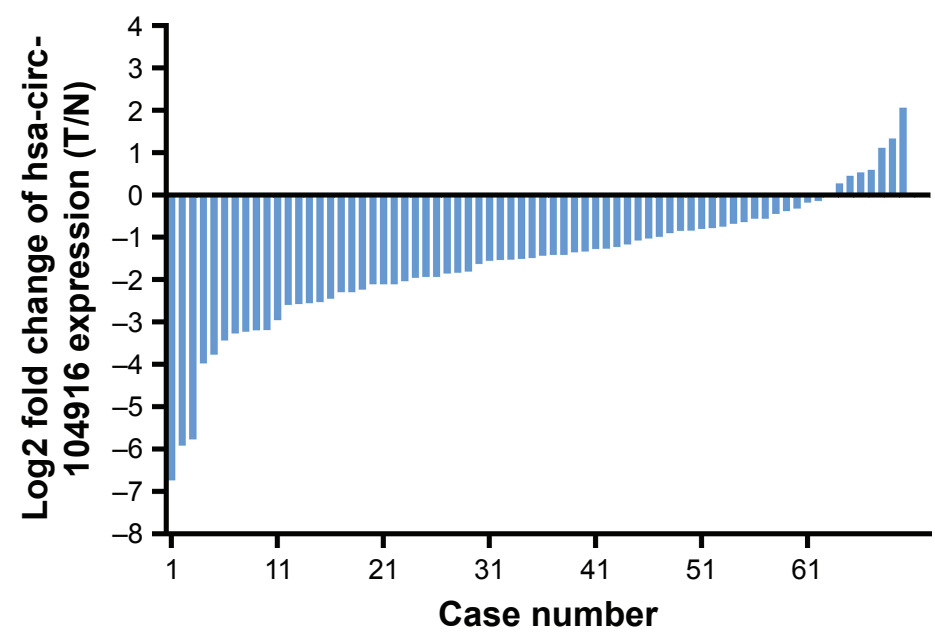

B

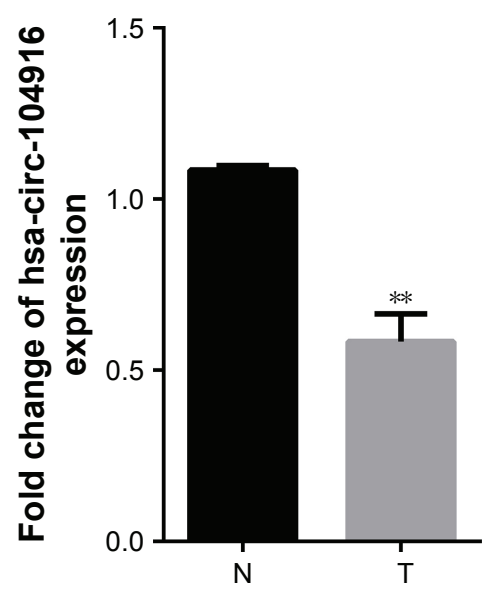

Figure I Circ-104916 expression in GC tissues.

Notes: (A) The expression of circ-104916 was validated by qPCR in seventy patients. Log2 fold changes in circ-104916 expressions (T/N) of each case are plotted. (B) The expression level of circ-104916 was significantly downregulated in tumor tissues. $* * P<0.01$.

Abbreviation: qPCR, quantitative polymerase chain reaction. 
tumor stage, lymphatic metastasis and nerve invasion (Table 1). However, there was no significant correlation between circ-104916 expression and other clinicopathological factors, such as age, histological grade and vascular invasion. Moreover, a lower expression of circ-104916 appeared in deeper invasion depth, higher tumor stage and more frequently lymphatic metastasis patients (Figure 2A-C). Taking together, our results showed that circ-104916 expression was associated with clinicopathological features and might be a potential biomarker of GC.

\section{Circ- 104916 suppresses proliferation of GC cells in vitro}

To detect the function of circ-104916 in the progression of GC, we performed gain of function assay. First, we analyzed circ-104916 expression in human GC cell lines. The expression of circ-104916 in AGS, MKN45, BGC823, MGC803 and 7901 was significantly lower than that in the normal gastric mucosa cell line GES-1 (Figure 3A), which was consistent with our clinical findings that human GC tissues had low levels of circ-104916. According to the endogenous expression of circ-104916 in five GC cell lines, we transfected pcDNA-hsa-circ-104916 or negative control in BGC823 and

Table I Correlation with the clinicopathological data and expression of circ-104916

\begin{tabular}{llll}
\hline Characteristics & Low & High & P-value \\
\hline Total & 35 & 35 & \\
Age (years) & & & 0.339 \\
$\quad<60$ & 15 & 20 & \\
$\quad \geq 60$ & 20 & 15 & \\
Sex & & & 0.045 \\
$\quad$ Male & 27 & 18 & \\
$\quad$ Female & 8 & 17 & \\
Differential status & & & 0.785 \\
$\quad$ Undifferentiated/poorly & 25 & 27 & \\
$\quad$ Moderate/well & 10 & 8 & \\
Nerve invasion & & & \\
$\quad$ Yes & 29 & 19 & \\
$\quad$ No & 6 & 16 & 0.019 \\
Vascular invasion & & & \\
$\quad$ Yes & 19 & 14 & \\
No & 16 & 21 & \\
T stages & & & \\
TI & 1 & 12 & 0.001 \\
T2-T4 & 34 & 23 & \\
N stage & & & \\
N0 & 6 & 16 & \\
NI-N3 & 29 & 19 & \\
TNM stages & & & \\
I-II & 9 & 20 & \\
III-IV & 26 & 15 & \\
\hline
\end{tabular}

MGC803 cell lines. After $48 \mathrm{~h}$ of treatment, the expression of circ-104916 was effectively increased (Figure 3B). Then, we assessed the effect of circ-104916 on the proliferation of GC cells though CCK-8 assay. The result showed that overexpression of circ-104916 could suppress the proliferation of BGC823 and MGC803 cell lines (Figure 3C). Our results indicated that circ-104916 was downregulated in GC cells and could inhibit the proliferation of GC cells.

\section{Circ- 104916 suppresses GC cell migration and invasion}

Migration and invasion assays were used to determine the change of migration and invasion capability of BGC823 and MGC803 cells, which were both transfected with pcDNAcirc-104916. The migration assay indicated that overexpression of circ-104916 significantly suppressed the migration capability of BGC823 and MGC803 cells (Figure 4A). Meanwhile, the invasion assay showed that overexpression of circ-104916 could inhibit the invasion capability of BGC823 and MGC803 cells (Figure 4B). These results suggested that circ-104916 could inhibit the migration and invasion of GC cells.

\section{Circ-1049I 6 suppresses GC cell invasion and migration via the epithelial- mesenchymal transition (EMT) process}

To further explore the underlying mechanism of decreased invasion and migration by circ-104916, EMT molecules were evaluated. The upregulation of E-cadherin was observed in circ-104916 overexpression of GC cells. Additionally, the downregulation of $\mathrm{N}$-cadherin and Vimentin were also observed in circ-104916 overexpression of GC cells. The decreased expression of Slug was accompanied with circ104916 overexpression (Figure 4C). In conclusion, our results suggested that circ-104916 might suppress GC cell migration and invasion via inhibiting the EMT process.

\section{Discussion}

In recent years, with the rapid development of RNA sequencing, researches have discovered that circRNA is abundant and especially expresses in a cell type or development stage. ${ }^{5}$ Circular RNAs can modulate cellular physiology as microRNA sponges. For instance, the circ-ITCH suppresses esophageal squamous cell carcinoma via the $\mathrm{Wnt} / \beta$-catenin pathway. ${ }^{15}$ Circ-001569 could promote proliferation and invasion of colorectal cancer via target mir-145. ${ }^{16}$ Previous studies also elaborated that circRNA could affect cell processes by forming RNA-protein complexes. ${ }^{17,18}$ For example, 
A

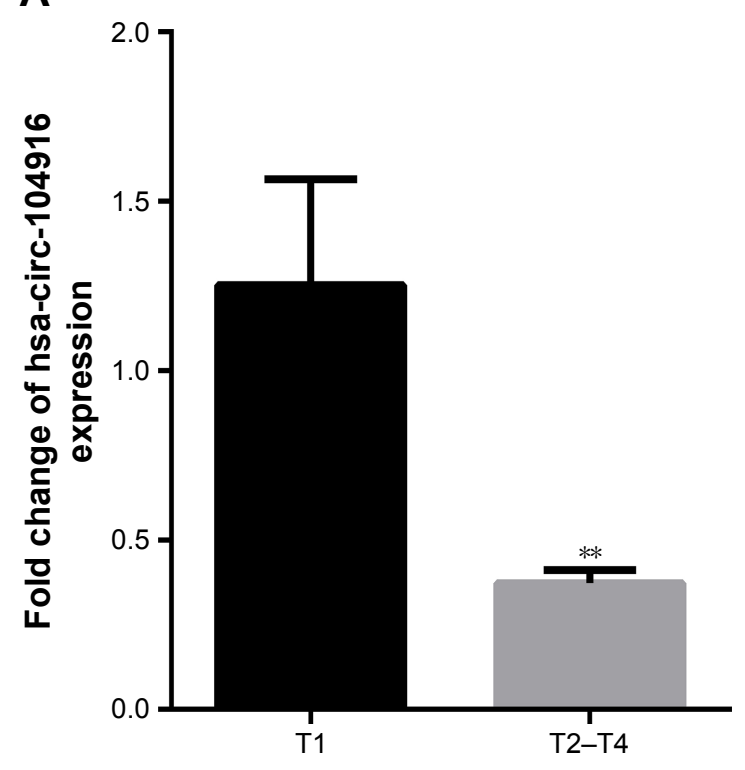

B

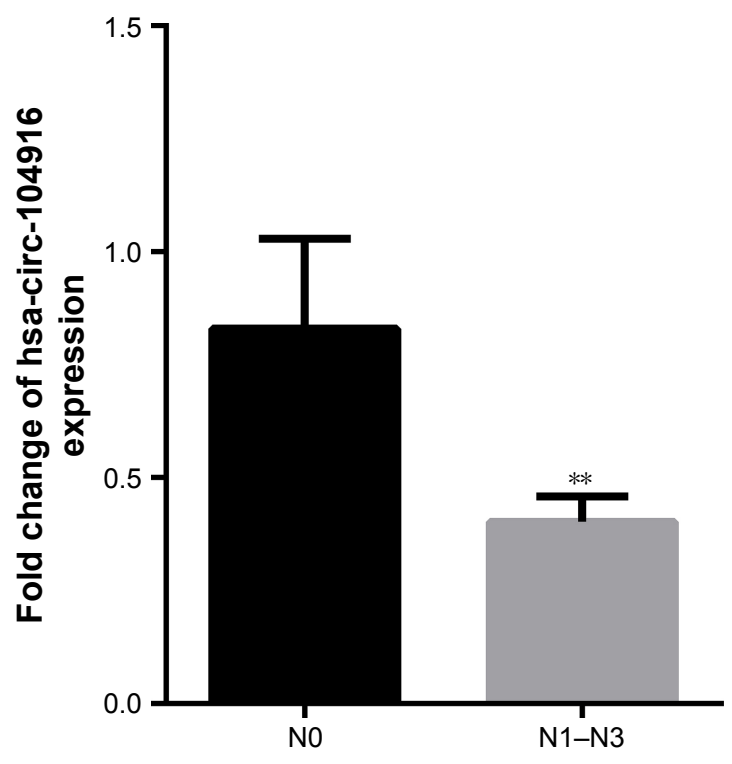

C

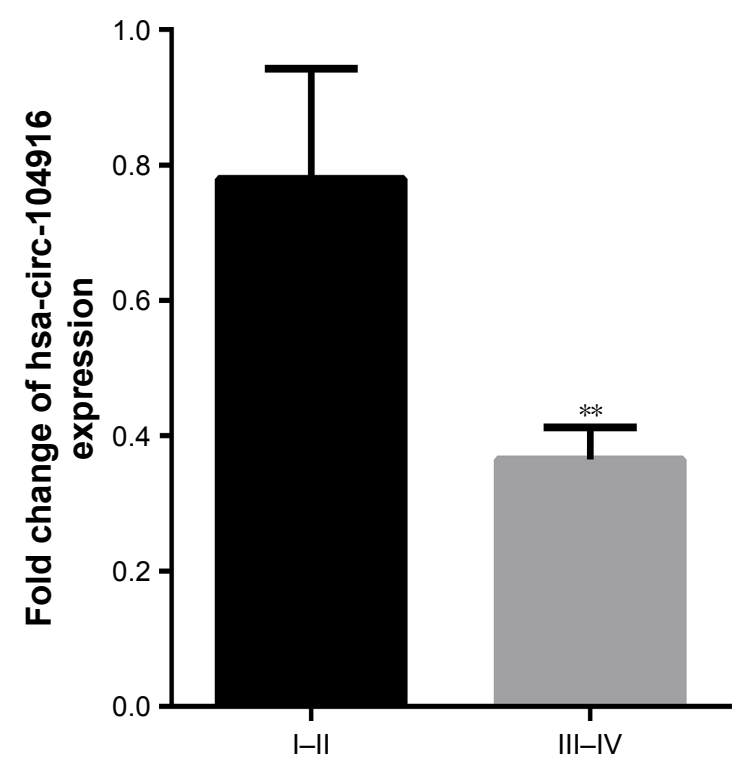

Figure 2 Clinicopathological factors with the expression level of circ-104916.

Notes: (A) The expression of circ- 104916 was much lower in the deeper invasion depth patients. (B) Circ- 104916 expression was downregulated in the patients with more frequent lymphatic metastasis. (C) The expression of circ-104916 was lower in higher tumor-stage patients. $* * P<0.01$.

Circ-Foxo3 not only could block cell-cycle progression via forming ternary complexes with $\mathrm{p} 21$ and $\mathrm{CDK} 2^{17}$ but also induces cell apoptosis via binding MDM2 and P53.18 Recent studies revealed that circular RNA could regulate transcription or splicing. Circular intronic RNA may have a cis-regulatory role on their parent coding genes. ${ }^{19}$ Thus, circRNA may be a core participant in physiology and pathology processes. To the best of our knowledge, there were few studies about circular RNA in GC. Only one study showed that circ-002059 in GC was significantly associated with
TNM stage and distal metastasis..$^{20}$ However, the effect and function of circRNA in GC remains unknown.

In our study, via microarray, we first found that circ104916 was significantly lower in the GC tissue than the adjacent normal tissues. Through database CircBase, ${ }^{21}$ we figured out that circ-104916 was generated from chromosomal region $9 \mathrm{q} 33.3$, which was back spliced by exon 1 , exon 3, exon 4, exon 5, exon 6 and exon 8 of NEK6. Mature circ-104916 transcript is a circular RNA molecule of 651 nt. Then, we validated the expression of circ-104916 


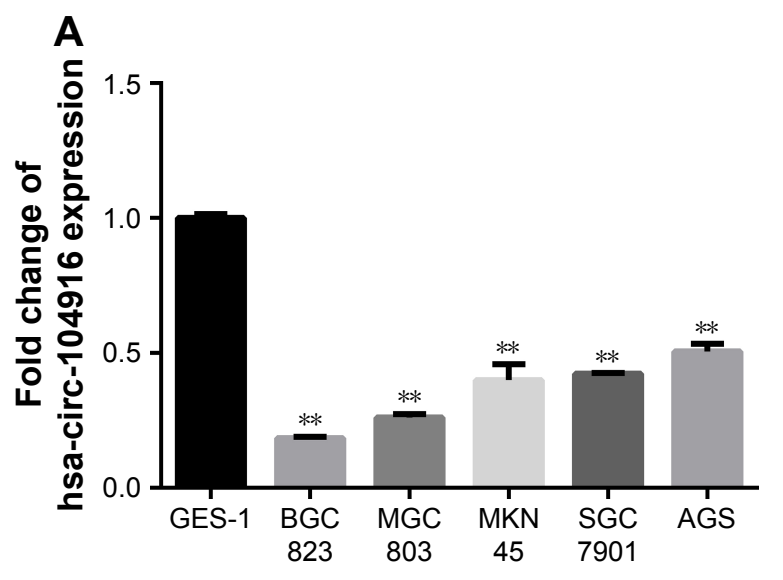

C

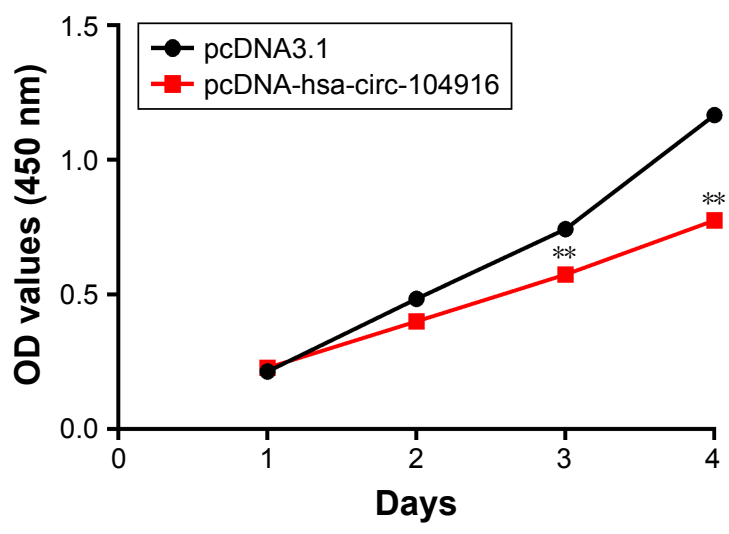

\section{B}

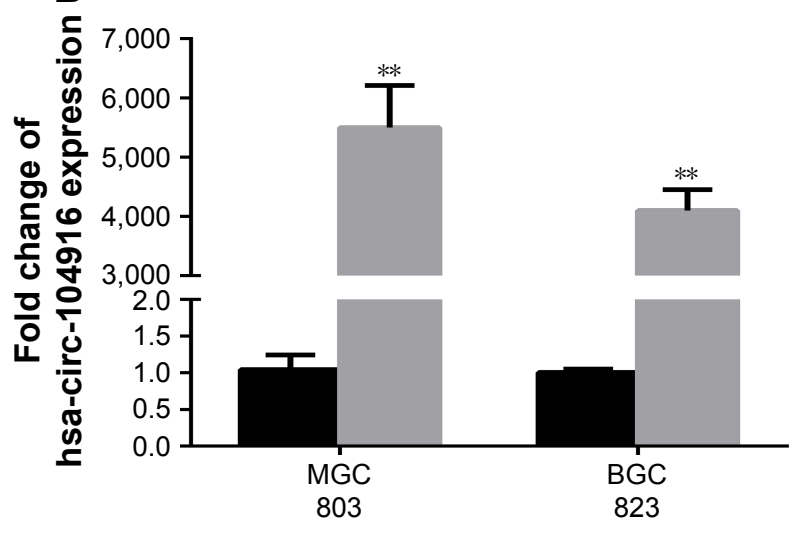

pcDNA3.1 pcDNA-hsa-circ-104916

MGC803

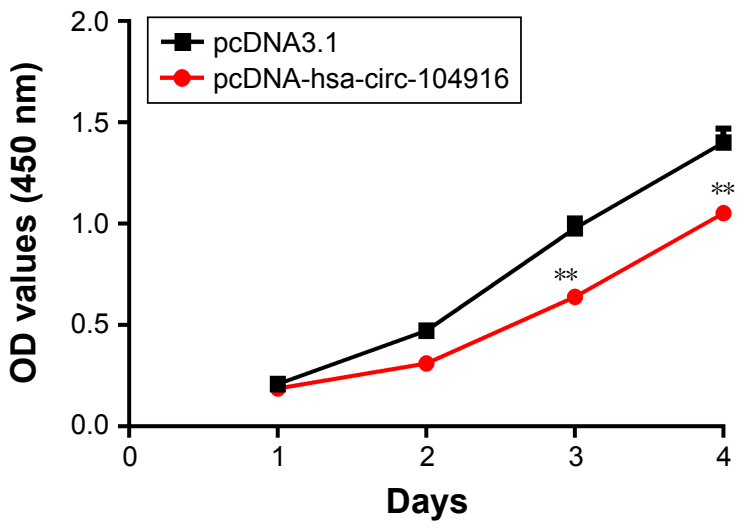

Figure 3 Circ-104916 overexpression suppressed the proliferation of GC cells.

Notes: (A) Circ-104916 was more downregulated in GC cell lines (BGC823, MGC803, MKN45, SGC790I, AGS) than in the normal gastric mucosa cell line (GES-I). (B) The effects of the pcDNA-hsa-circ-104916 plasmid on the expression of circ-104916 in GC cell lines. (C) Effect of circ-104916 on cell proliferation in vitro by CCK-8 assay. Error bars represent mean \pm SEM from three independent experiments. $* * P<0.01$.

Abbreviations: GC, gastric cancer; CCK-8, Cell Counting Kit-8; OD, optical density; SEM, standard error of the mean.

in 70 matched samples. The result was coincident with the microarray finding. Moreover, deeper invasion depth, higher tumor stage and more frequent lymphatic metastasis patients had a lower expression of circ-104916. These results indicated that circ-104916 might be a potential biomarker for GC.

In in vitro experiments, the results suggested that circ104916 could suppress the proliferation, migration and invasion abilities of GC cells. Therefore, circ-104916 may be a potential tumor suppressor and play a core role in $\mathrm{GC}$ invasion and metastasis. EMT is a critical cellular progress in cancer metastasis. ${ }^{22}$ What is more, a previous study had reported that hundreds of circRNAs were regulated during human EMT..$^{23}$ The key events in EMT are dissolution of the epithelial cell-cell junction, downregulation of an epithelial gene expression signature and activation of genes that help to define the mesenchymal phenotype and increased cell protrusions and motility. ${ }^{24}$ By Western blot, we found that the epithelial molecule (E-cadherin) was upregulated and the mesenchymal molecule (N-cadherin, Vimentin) was downregulated after circ-104916 overexpression. Slug (also known as Snail2) is a member of the SNAIL family of zinc finger transcriptional repressors, which can repress the E-cadherin expression. ${ }^{25}$ In our data, circ-104916 could decrease Slug expression. Taking together, the results suggested that circ104916 might have epithelial-specific functions through decreasing the Slug expression.

Our study has several limitations. First, we did not perform loss of function because of the low efficiency of siRNA resulting from the high GC rate around the back-splice sites. Second, these conclusions were based on the responses of two cell lines and may not reflect the process in the organism. The conformance in mice needs to be accomplished in the future.

In summary, our data first found that circ-104916 was downregulated in GC tissues and the expression of 
A

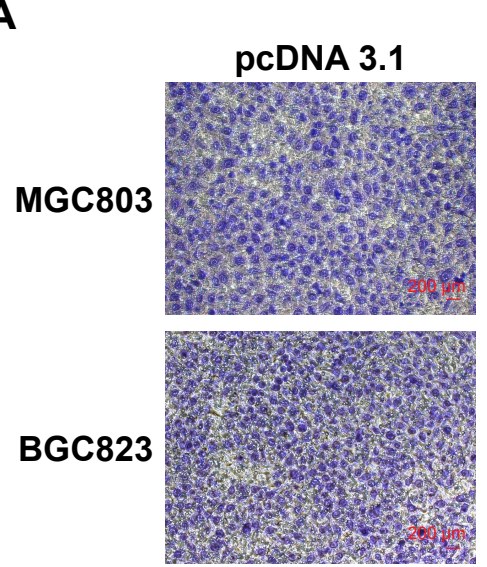
pcDNA-hsa-

\section{circ-104916}

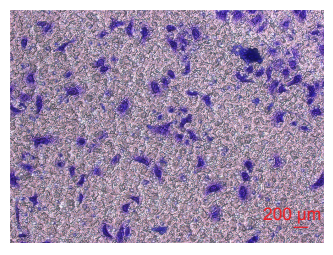

B
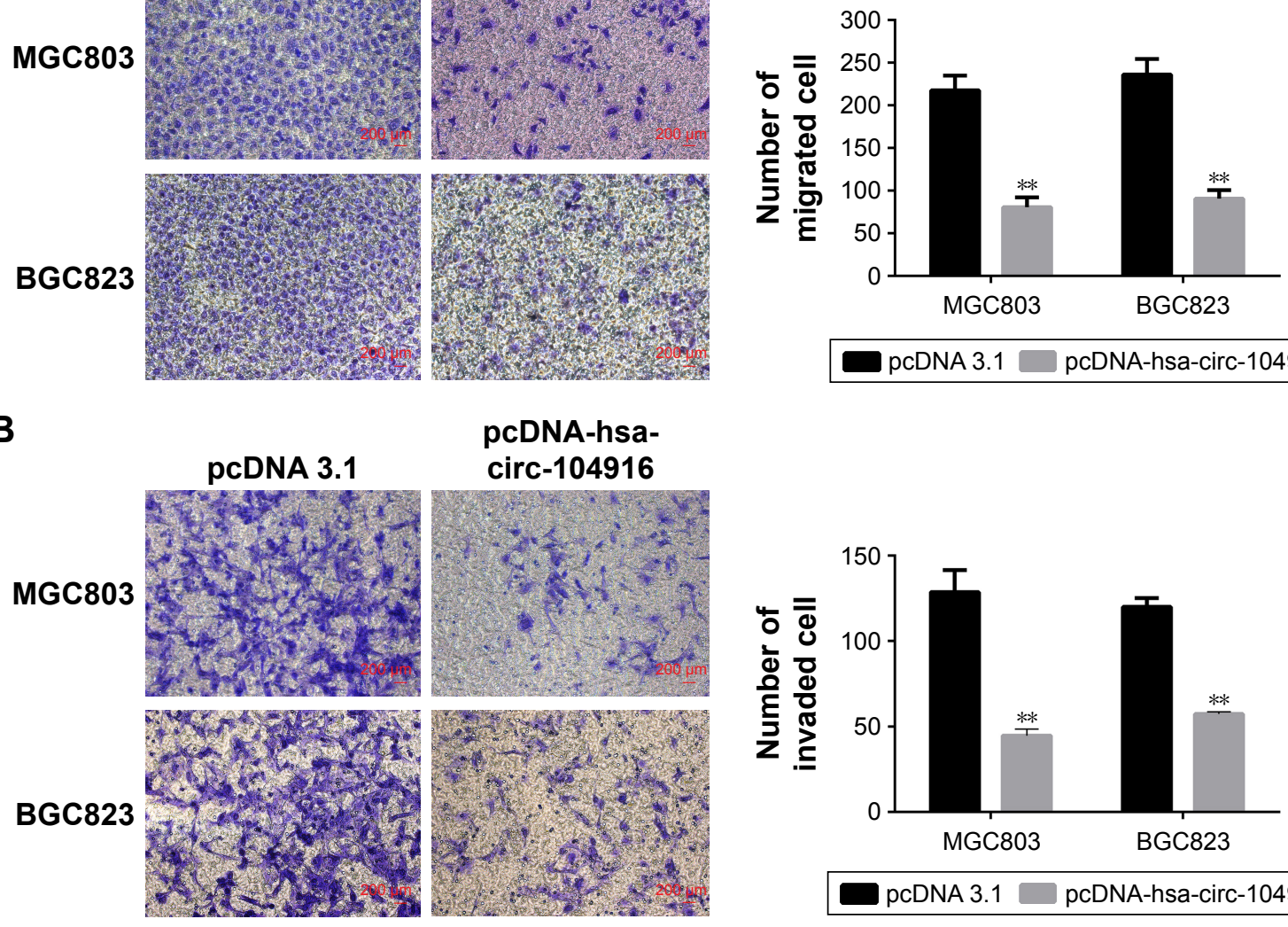

pcDNA-hsa-

$$
\text { circ-104916 }
$$
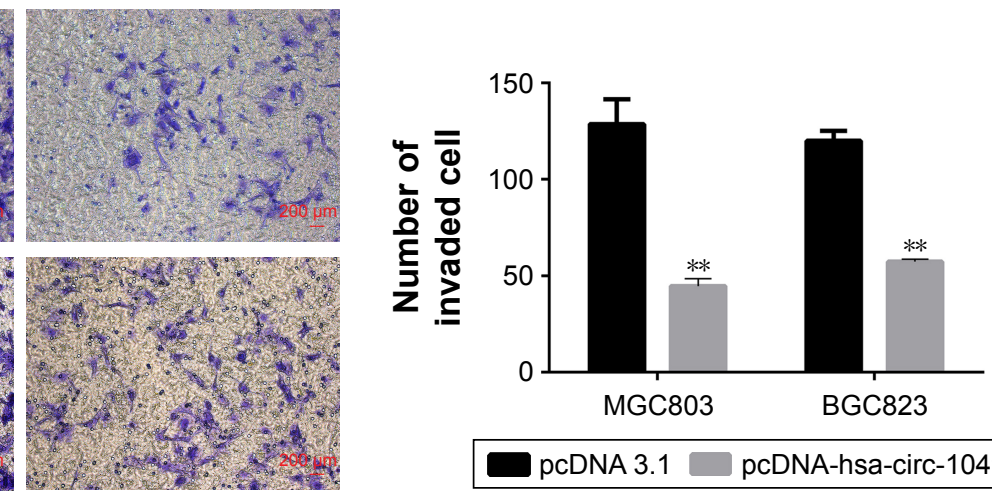

C

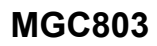

\section{BGC823}

pcDNA-circ-104916
pcDNA 3.1

E-cadherin

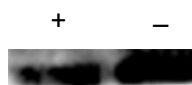

$\mathrm{N}$-cadherin

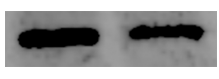

Vimentin

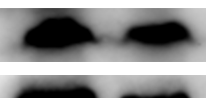

Slug

GAPDH
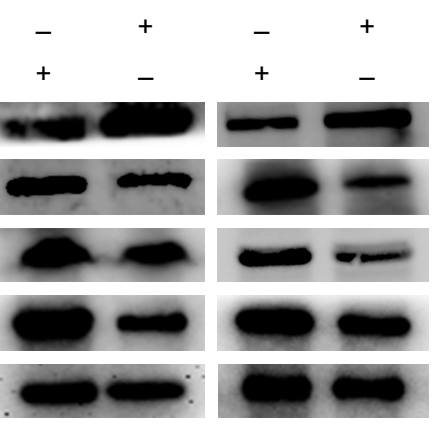

Figure 4 Circ-104916 inhibited the migration and invasion of GC cells.

Notes: (A) After transfecting pcDNA-hsa-circ-0067934, GC cells showed reduced migration in transwell migration assays. (B) Circ-1049I6 also suppressed the invasion ability of BGC823 and MGC803 cells. $* * P<0.01$. (C) After transfecting pcDNA-hsa-circ-104916, the protein level of key molecules of EMT was assessed by Western blot. Abbreviations: GC, gastric cancer; EMT, epithelial-mesenchymal transition.

circ-104916 was related to invasion depth, tumor stage and lymphatic metastasis. The overexpression of circ-104916 suppressed the migration and invasion of GC cells through altering the EMT process. Our research suggested that circ10496 might be a novel potential tumor suppressor and biomarker of GC.

\section{Acknowledgments}

The support of Zhen Han in data analysis was essential in this paper. This work was supported by the grants from
Guangdong Provincial Science and Technology Key Project (2014A020215014), the Research Fund of Public Welfare in the Health Industry, the National Health and Family Planning Commission of China (201402015) and the Key Clinical Specialty Discipline Construction Program, National Natural Science Foundation of China (81672446 and 81600510).

\section{Disclosure}

The authors report no conflicts of interest in this work. 


\section{References}

1. Jemal A, Bray F, Center MM, Ferlay J, Ward E, Forman D. Global cancer statistics. CA Cancer J Clin. 2011;61(2):69-90.

2. Hu Y, Huang C, Sun Y, et al. Morbidity and mortality of laparoscopic versus open D2 distal gastrectomy for advanced gastric cancer: a randomized controlled trial. J Clin Oncol. 2016;34(12):1350-1357.

3. Qi X, Liu Y, Wang W, et al. Management of advanced gastric cancer: an overview of major findings from meta-analysis. Oncotarget. 2016; 7(47):78180-78205

4. Sanger HL, Klotz G, Riesner D, Gross HJ, Kleinschmidt AK. Viroids are single-stranded covalently closed circular RNA molecules existing as highly base-paired rod-like structures. Proc Natl Acad Sci US A. 1976; 73(11):3852-3856.

5. Memczak S, Jens M, Elefsinioti A, et al. Circular RNAs are a large class of animal RNAs with regulatory potency. Nature. 2013;495(7441): 333-338.

6. Qi X, Zhang DH, Wu N, Xiao JH, Wang X, Ma W. ceRNA in cancer: possible functions and clinical implications. J Med Genet. 2015;52(10): 710-718.

7. Qu S, Song W, Yang X, et al. Microarray expression profile of circular RNAs in human pancreatic ductal adenocarcinoma. Genom Data. 2015; 5:385-387.

8. Ahmed I, Karedath T, Andrews SS, et al. Altered expression pattern of circular RNAs in primary and metastatic sites of epithelial ovarian carcinoma. Oncotarget. 2016;7(24):36366-36381.

9. Zhong Z, Lv M, Chen J. Screening differential circular RNA expression profiles reveals the regulatory role of circTCF25-miR-103a-3p/ miR-107-CDK6 pathway in bladder carcinoma. Sci Rep. 2016; 6:30919.

10. Shang X, Li G, Liu H, et al. Comprehensive circular RNA profiling reveals that hsa_circ_0005075, a new circular RNA biomarker, is involved in hepatocellular carcinoma development. Medicine (Baltimore). 2016;95(22):e3811.

11. Hansen TB, Jensen TI, Clausen BH, et al. Natural RNA circles function as efficient microRNA sponges. Nature. 2013;495(7441):384-388.
12. Xu L, Zhang M, Zheng X, Yi P, Lan C, Xu M. The circular RNA ciRS-7 (Cdrlas) acts as a risk factor of hepatic microvascular invasion in hepatocellular carcinoma. J Cancer Res Clin Oncol. 2017;143(1):17-27.

13. Yu L, Gong X, Sun L, Zhou Q, Lu B, Zhu L. The circular RNA Cdr1as act as an oncogene in hepatocellular carcinoma through targeting miR-7 expression. PLoS One. 2016;11(7):e0158347.

14. Zhang Y, Li J, Yu J, et al. Circular RNAs signature predicts the early recurrence of stage III gastric cancer after radical surgery. Oncotarget. 2017;8(14):22936-22943.

15. Li F, Zhang L, Li W, et al. Circular RNA ITCH has inhibitory effect on ESCC by suppressing the Wnt/ $\beta$-catenin pathway. Oncotarget. 2015; 6(8):6001-6013.

16. Xie H, Ren X, Xin S, et al. Emerging roles of circRNA_001569 targeting miR-145 in the proliferation and invasion of colorectal cancer. Oncotarget. 2016;7(18):26680-26691.

17. Du WW, Yang W, Liu E, Yang Z, Dhaliwal P, Yang BB. Foxo3 circular RNA retards cell cycle progression via forming ternary complexes with p21 and CDK2. Nucleic Acids Res. 2016;44(6):2846-2858.

18. Du WW, Fang L, Yang W, et al. Induction of tumor apoptosis through a circular RNA enhancing Foxo3 activity. Cell Death Differ. 2017;24(2):357-370.

19. Zhang $\mathrm{Y}$, Zhang XO, Chen T, et al. Circular intronic long noncoding RNAs. Mol Cell. 2013;51(6):792-806.

20. Li P, Chen S, Chen H, et al. Using circular RNA as a novel type of biomarker in the screening of gastric cancer. Clin Chim Acta. 2015;444: 132-136.

21. Glažar P, Papavasileiou P, Rajewsky N. circBase: a database for circular RNAs. RNA. 2014;20(11):1666-1670.

22. Nieto MA. Epithelial plasticity: a common theme in embryonic and cancer cells. Science. 2013;342(6159):1234850.

23. Conn SJ, Pillman KA, Toubia J, et al. The RNA binding protein quaking regulates formation of circRNAs. Cell. 2015;160(6):1125-1134.

24. Qi X, Zhang L, Lu X. New insights into the epithelial-to-mesenchymal transition in cancer. Trends Pharmacol Sci. 2016;37(4):246-248.

25. Hajra KM, Chen DY, Fearon ER. The SLUG zinc-finger protein represses E-cadherin in breast cancer. Cancer Res. 2002;62(6):1613-1618. 


\section{Supplementary material}

$\begin{array}{lllllllllllll}\text { T1 } & \text { T2 } & \text { T3 } & \text { T4 } & \text { T5 } & \text { T6 } & \text { N1 } & \text { N2 } & \text { N3 } & \text { N4 } & \text { N5 } & \text { N6 }\end{array}$

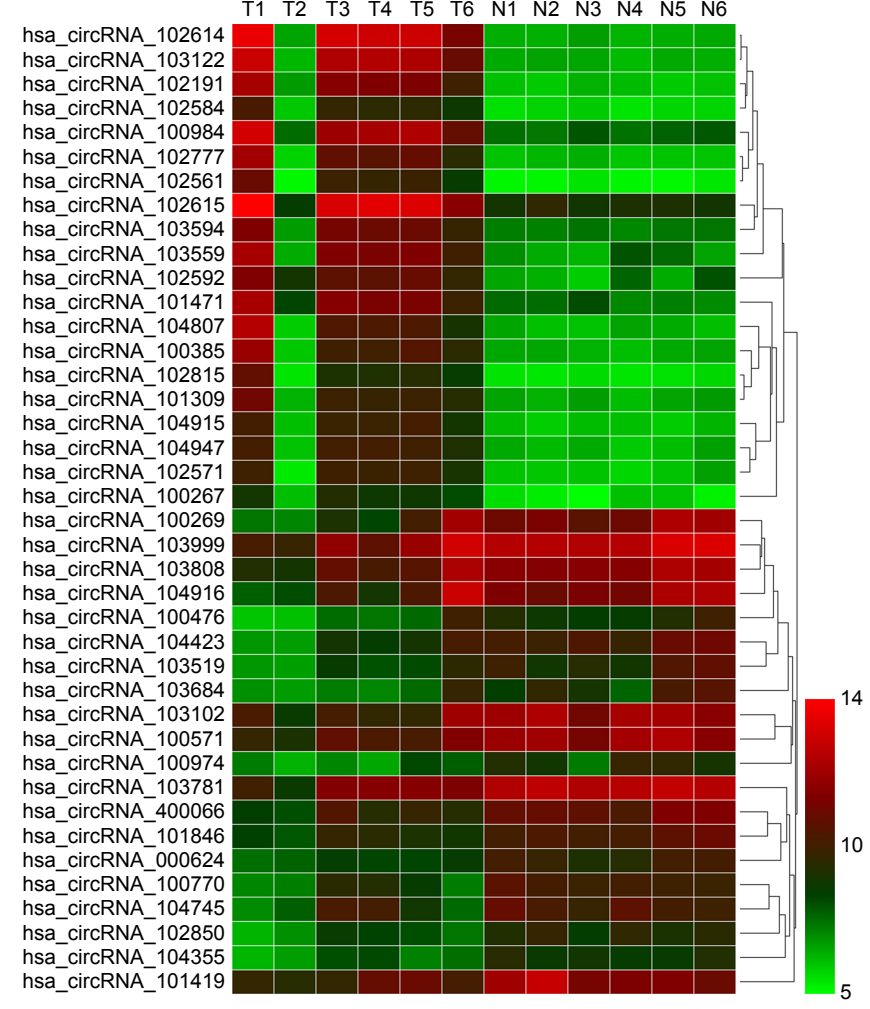

Figure SI Hierarchical clustering analysis of the most up- and down regulated circRNAs.

Notes: Each column represents a sample, and each row represents a circRNA. Red strip represents a high relative expression, and green strip represents a low relative expression. T represents $\mathrm{GC}$ tissues and $\mathrm{N}$ represents normal gastric mucosa.

Abbreviation: GC, gastric cancer.

\section{Publish your work in this journal}

OncoTargets and Therapy is an international, peer-reviewed, open access journal focusing on the pathological basis of all cancers, potential targets for therapy and treatment protocols employed to improve the management of cancer patients. The journal also focuses on the impact of management programs and new therapeutic agents and protocols on

\section{Dovepress}

patient perspectives such as quality of life, adherence and satisfaction. The manuscript management system is completely online and includes a very quick and fair peer-review system, which is all easy to use. Visit http://www.dovepress.com/testimonials.php to read real quotes from published authors. 